\title{
Lernen im freiwilligen Engagement und gesellschaftliche Partizipation
}

\section{Ein empirischer Versuch zur Erklärung politischen Handelns}

\author{
Gerald Prein · Erich Sass • Ivo Züchner
}

Zusammenfassung: Der Beitrag untersucht die Frage, ob und in welchem Maße sich freiwilliges Engagement in der Jugend auf tatsächliche politische Partizipation im Erwachsenenalter auswirkt. Er nimmt theoretischen Bezug auf Deweys Theorie demokratischer Erziehung sowie auf die Theorie gemeinnütziger Tätigkeit von Reinders. Dabei steht die Frage im Vordergrund, inwieweit der Erwerb von spezifischen Kompetenzen und Wissen im Umgang mit sozialen und politischen Institutionen für späteres politisches Engagement genauso oder sogar bedeutsamer sind als die Herausbildung und Weiterentwicklung prosozialer Eigenschaften. Basierend auf der Datengrundlage einer Untersuchung, in der in der Jugend freiwillig Engagierte sowie eine Kontrollgruppe Nichtengagierter (Gesamt $n=2.052$ ) befragt wurden, wird dieser Zusammenhang auf der Grundlage ordinaler Probitmodelle untersucht. Hierbei zeigt sich, dass es neben Einstellungsveränderungen spezifische Handlungserfahrungen im freiwilligen Engagement sind, die eine politische Partizipation fördern. Politisches Engagement ist damit ganz wesentlich mit Lern- und Bildungsprozessen im Deweyschen Sinne verbunden.

Schlüsselwörter: Jugend · Freiwilliges Engagement · Ehrenamt · Politisches Handeln · Demokratische Erziehung · Lernen

Online publiziert: 28.08 .2009

(C) VS-Verlag 2009

Dr. G. Prein $(\bowtie)$

Deutsches Jugendinstitut, Nockherstr. 2, 81541 München, Deutschland

E-Mail: prein@dji.de

E. Sass, M.A.

Forschungsverbund Deutsches Jugendinstitut e.V./TU Dortmund

Vogelpothsweg 78, 44227 Dortmund, Deutschland

E-Mail: ESass@fk12.tu-dortmund.de

Dr. I. Züchner

Deutsches Institut für Internationale Pädagogische Forschung

Schloßstraße 29, 60486 Frankfurt a.M., Deutschland

E-Mail: zuechner@dipf.de 


\title{
Learning in voluntary work and social participation - An empirical attempt to explain political action
}

\begin{abstract}
This contribution analyses the impact of young people's work on political participation in adulthood on the basis of Dewey's theory of democratic education and a theory of community service sketched by Reinders. Its primary goal is to examine whether the acquisition of specific skills and specific knowledge obtained in social and political institutions is as important for - or even more important than - future political commitment as the development of pro-social attitudes. Based on survey data $(n=2,052)$, these assumptions are tested using ordinal probit models. These models show that in addition to effects of changes in attitude, specific experiences in voluntary work seem to be important to promoting political participation. Political commitment is, therefore, indeed closely connected to learning and educational processes in the sense of Dewey.
\end{abstract}

Keywords: Community service $\cdot$ Democratic education $\cdot$ Learning $\cdot$ Political action $\cdot$ Volunteering · Youth

\section{Einleitung}

Wie werden eigentlich Demokraten gemacht? Wo können Jugendliche lernen, gesellschaftlich zu partizipieren und politisch zu handeln? Nicht erst seit der Debatte um die Ergebnisse der PISA-Studie (vgl. Baumert et al. 2001) ist bekannt, dass das formale Bildungssystem nicht ausreicht, um Heranwachsende auf die Anforderungen einer globalisierten Gesellschaft vorzubereiten. Dies gilt für ihre berufliche Orientierung, aber auch - und darum soll es im Folgenden gehen - für ihr Hineinwachsen in das demokratische Gemeinwesen.

Wenngleich Bildung und Lernen Jugendlicher von Politik und Öffentlichkeit immer noch vorrangig der Schule zugeordnet werden, werden im aktuellen Diskurs zunehmend außerschulische, häufig als ,informell“ bezeichnete Lernfelder in den Blick genommen (vgl. Dohmen 2001; Münchmeier, Otto \& Rabe-Kleberg 2002; Furtner-Kallmünzer et al. 2002; Brenner 2003; Otto \& Rauschenbach 2004; Konsortium Bildungsberichterstattung 2006; Autorengruppe Bildungsberichterstattung 2008). Als ein „informelles“ Lernfeld mit besonderer Bedeutung für die Reproduktion der Demokratie erscheint dabei das freiwillige Engagement. In den bildungspolitischen Debatten führen Vertreter der Verbände, aber auch der Politik und der Wissenschaft dieses Feld gerne als einen außerschulischen Lernort an, in dem Lern- und Bildungsprozesse, insbesondere sozialer Art, gemacht werden und das Hineinwachsen in demokratische Spielregeln unterstützt wird (vgl. Thole \& Hoppe 2003; Enquete-Kommission „Zukunft des bürgerschaftlichen Engagements“ des Deutsches Bundestags 2002, S. 552 ff.; Corsa 1998, 2003; Olk 2003; Buhl \& Kuhn 2005). Freiwilliges Engagement - so die allgemeine Grundüberzeugung - mit seinen unterschiedlichen Möglichkeiten und Graden der Verantwortungsübernahme, der Mitgestaltung und der aktiven Teilnahme schafft Gelegenheiten für vielfältige Lern-, Bildungsund Entwicklungsprozesse, die sich, vor allem aufgrund der Freiwilligkeit der Teilnahme, von den Lernorten des formalen Bildungssystems grundlegend unterscheiden.

Allerdings beruht diese mit großem Konsens geteilte Annahme nicht auf empirisch fundierten Analysen, sondern eher auf persönlicher Erfahrung und Anschauung der 
Beteiligten. Ob freiwilliges Engagement in der Jugendzeit tatsächlich maßgeblich und ursächlich an der Erzeugung nachhaltigen demokratischen Bewusstseins und politischen Handelns beteiligt ist, ist bislang nicht nachgewiesen. Die Bereitschaft, sich im Jugendalter zu engagieren, könnte ebenso wie die Bereitschaft zur gesellschaftlichen Partizipation im Erwachsenenalter von Faktoren abhängen, die anderen Ursprungs sind, etwa das Aufwachsen in der Familie oder die politische Bildung in der Schule.

\section{Forschungsstand}

Wenngleich die bundesdeutsche Forschung zum freiwilligen Engagement seit Mitte der 1980er-Jahre einen (mittlerweile wieder abklingenden) Boom zu verzeichnen hat (vgl. zusammenfassend Düx 1999; Enquete-Kommission 2002), fällt auf, wie wenig bisher Bildungs- und Lernprozesse im freiwilligen Engagement in den Blick der Forschung genommen wurden. Insbesondere in den beiden Freiwilligensurveys (vgl. Bundesministerium für Familie, Senioren, Frauen und Jugend 2000; vgl. Gensicke, Picot \& Geiss 2006) oder auch in Überblicksstudien, wie dem Sozio-ökonomischen Panel (vgl. etwa Kühnemund \& Schupp 2007) oder der Zeitbudgeterhebung 2001/2 (vgl. Bundesministerium für Familie, Senioren, Frauen und Jugend \& Statistisches Bundesamt 2003), finden sich empirische Aussagen zum Umfang, zur Dauer, zu Tätigkeitsfeldern und Motiven oder auch zur Qualifikation, sozialen Integration und Engagementbereitschaft Jugendlicher, aber nur wenig zu Lerneffekten und zu Auswirkungen auf die spätere Partizipationsbereitschaft. Dies gilt auch für Jugendstudien, wie dem Jugendsurvey (zuletzt Gille et al. 2006) oder die jüngeren ShellStudien (Jugendwerk der Deutschen Shell 1997, 2000; Hurrelmann \& Albert 2002, 2006).

Auch in kleineren, regional begrenzten oder auf Einzelorganisationen bezogene Studien zum freiwilligen Engagement Jugendlicher finden sich nur ansatzweise Aussagen zu Lernprozessen, zum Kompetenzerwerb und zur Wirkung auf die Bereitschaft zur politischen Teilhabe im Erwachsenenalter (vgl. etwa Reichwein \& Freund 1992; Auerbach \& Wiedemann 1997; im Überblick Düx 1999). Erst in der jüngeren Jugendverbandsforschung werden auch Fragen des Kompetenzerwerbs, des sozialen Lernens und der Nachhaltigkeit im Engagement erworbener Fähigkeiten aufgeworfen (vgl. Fischer 2001; Lehmann 2004; Schwab 2006; Richter, Jung \& Riekmann 2006; Fauser, Fischer \& Münchmeier 2006). Die genannten Studien aus der Jugendverbandsforschung gewinnen ihre Daten in erster Linie aus Untersuchungen bei Jugendlichen (Ausnahme Schwab, der ehemalige Jugendleiter/-innen befragt hat), sodass zwar Aussagen zur Kompetenz- und Persönlichkeitsentwicklung getroffen werden können, aber nur wenig über die Nachhaltigkeit des im Engagement Erlernten ausgesagt werden kann.

Die Frage der Erfassung und Sichtbarmachung der in einem freiwilligen Engagement informell erworbenen Kompetenzen versuchen Gerzer-Sass, Reupold und Nußhart (2006) mit dem Instrument der Kompetenzbilanz zu beantworten. Hierbei handelt es sich um ein Instrument der Selbst- und Fremdeinschätzung, nicht der empirischen Analyse.

Aus dem internationalen Kontext hatten, neben der internationalen Vergleichsstudie zum Engagement von Gaskin et al. (1996), die Arbeiten Putnams (1993, 2000) zum Sozialkapital besonderen Einfluss auf die Diskussion in Deutschland. Mit der besonderen Frage des Zusammenhangs von Engagement, politischem Wissen und politischer Betei- 
ligungsbereitschaft hat sich die sogenannte Civic-Education-Studie der IEA (International Association for the Evaluation of Educational Achievement) beschäftigt. Hier wurde ein für alle 28 teilnehmenden Länder verbindlicher Test entwickelt, mit dem ermittelt werden sollte, in welchem Maße Jugendliche demokratische Prinzipien und Prozesse kennen und verstehen. Ein wichtiges Ergebnis dieser Studie war, dass für das politische Verstehen und die gesellschaftliche Engagementbereitschaft Jugendlicher eine praktizierte demokratische Teilhabe in der Schule und in Jugendorganisationen wichtig sind (vgl. Torney-Purta et al. 2001; Oser \& Biedermann 2003). Die deutsche Teilstudie der Civic-Education-Studie hat ergeben, dass deutsche Schülerinnen und Schüler ein durchschnittliches politisches Wissen und ein positives Verhältnis zur Demokratie haben, im internationalen Vergleich allerdings politisch weniger handlungs- und engagementbereit sind (vgl. Oesterreich 2002). Einen Überblick zum Stand jüngster internationaler, vor allem US-amerikanischer Forschung liefert Lerner (2007). Auch hier wird auf den relativ geringen Einfluss rein schulischen Lernens auf die Partizipationsbereitschaft hingewiesen. Effizienter (auch kosteneffizienter) erscheinen nach diesen Studien integrative Programme, welche die Beteiligung an sinnvollen Gemeinschaftsarbeiten und außerschulischem Engagement mit schulischer Wissensvermittlung kombinieren.

Darüber hinaus gibt es vor allem im anglo-amerikanischen Raum Forschungsansätze, welche die Relevanz des jugendlichen Engagements für eine demokratische Bürgergesellschaft in den Blick nehmen. In amerikanischen Längsschnittstudien konnten positive Zusammenhänge zwischen sozialem Engagement im Jugendalter und sozialem Engagement sowie Wahlbeteiligung im Erwachsenenalter nachgewiesen werden (vgl. Verba et al. 1995; Youniss et al. 1997). Yates und Youniss (1996) stellen in einer empirischen Studie zum sozialen Engagement Jugendlicher in den USA fest, dass freiwilliges Engagement in öffentlichen Kontexten persönliche Lern- und Entwicklungsprozesse insbesondere dort fördern kann, wo die im Engagement übernommenen Aufgaben, etwa die Betreuung sozial benachteiligter Personen, zur Reflexion der gesellschaftlichen Verhältnisse anregen.

$\mathrm{Zu}$ ähnlichen Befunden gelangt Hofer (1999) in Deutschland mit einer empirischen Untersuchung engagierter Jugendlicher in der Freiwilligen Feuerwehr und des Roten Kreuzes. Hofer und Buhl (2000) kommen bei der Sichtung empirischer Studien zum Einfluss freiwilligen Engagements auf die Persönlichkeitsentwicklung Jugendlicher zu dem Befund, dass trotz der Heterogenität der Forschungsergebnisse von positiven Einflüssen sozialen Engagements auf die Persönlichkeitsentwicklung ausgegangen werden kann.

Reinders (2005) hat eine empirische Studie zu Wertvorstellungen, Zukunftsperspektiven und sozialem Engagement von 13- bis 17-jährigen Schülerinnen und Schülern durchgeführt. Er weist nach, dass soziales Engagement als dritte Säule neben Eltern und Peers einen substanziellen Beitrag zu Werteentwicklung und prosozialem Verhalten Jugendlicher leistet. Einen weiterführenden Beitrag zu dieser Debatte stellt der 2006 in der Zeitschrift für Erziehungswissenschaft erschienene Artikel von Reinders (2006) dar. Da sich der Beitrag um eine Erweiterung dieser Perspektive bemüht, wird im Folgenden neben dem eigenen theoretischen Bezugsrahmen auch der Ansatz von Reinders dargestellt. 


\section{Theoretische Überlegungen zum Lernen im freiwilligen Engagement}

Den zentralen theoretischen Anknüpfungspunkt für die folgenden Überlegungen bietet Deweys Ansatz des „learning by doing“ und der demokratischen Erziehung. Lernen findet nach Dewey durch aktive Erfahrung und Reflexion statt: „Ein Gramm Erfahrung ist besser als eine Tonne Theorie, einfach nur deswegen, weil jede Theorie nur in der Erfahrung lebendige und der Nachprüfung zugängliche Bedeutung hat." (Dewey 2000, S. 193) Dabei kommt den Erfahrungen eine zentrale Funktion zu, da jede Erfahrung alle nachfolgenden in ihrer Qualität modifiziert und zugleich selbst geprägt ist durch vorangegangene Erfahrungen (vgl. Nohl 2001, S. 609). Dewey unterstreicht mit seiner Betonung der Erfahrung und Reflexion zugleich die Bedeutung der Ernstsituation für den Lernprozess. Die Realität der Erfahrungen, die Erfahrbarkeit der Konsequenzen beinhaltet die Grundlage des Lernens. Der Lerneffekt entsteht nach Dewey vor allem dadurch, dass bei Handelnden in der Situation auch die Notwendigkeit entsteht, über die Konsequenzen der Lösung nachzudenken, um notfalls korrigierend handeln zu können (vgl. Oelkers 2000, S. 337). Das Betrachten der Konsequenzen, die Reflexion des Lösungsansatzes wird zum zentralen Element des Lernens (vgl. Dewey 2000, S. 187). Der Reflexionsprozess beginnt mit einer Beunruhigung, einem Staunen, einem Zweifel und beschreibt das „Bemühen, zwischen unserem Handeln und seinen Folgen die Beziehungen im Einzelnen aufzudecken, so dass die beiden zu einem Zusammenhange verschmelzen" (Faulstich 2005, S. 531).

Dies gilt insbesondere für das Lernen von Demokratie, das im Zentrum vieler Arbeiten von Dewey steht. Demokratie begreift Dewey nicht nur als eine Form politischer Organisation, sondern als Lebensform. Im Rahmen einer demokratischen Pädagogik entwirft er einen Ansatz, um demokratische Methoden in Schul- und Unterrichtsorganisation zu integrieren. Dewey versteht „Demokratie“ nicht nur oder nicht primär als Lerninhalt, sondern vorrangig auch als Form aktiven Lernens: „Soziale Auffassungen und Interessen können nur in einer echt sozialen Umwelt entwickelt werden, in einer Umwelt, wo eine gemeinsame Erfahrung im wechselseitigen Geben und Nehmen aufgebaut wird." (Dewey 2000, S. 457)

Die Lernanlässe, die dem freiwilligen Engagement innewohnen, entsprechen in vielem dem von Dewey skizzierten praktischen Lernen. So bedeutet ehrenamtliches Engagement - egal, ob temporär oder lang anhaltend, in Initiativen, Vereinen oder Verbänden Handeln und Entscheiden in Alltagssituationen, die für die Jugendlichen Ernstcharakter haben. Jugendliche sind im ehrenamtlichen Engagement, das in der Regel in sozialen Kontexten (z.B. im Jugendverband, in der Schule, in Vereinen oder Kirchengemeinden) stattfindet, mit Handlungsanforderungen und der Notwendigkeit konfrontiert, Entscheidungen zu fällen, deren Folgen für sie erfahrbar bleiben. Handlungen und Wirkungen von Handlungen sind im Ehrenamt miteinander verwoben. Mit dem ehrenamtlichen Engagement stehen Jugendliche zudem vielfach vor der Aufgabe, eigene Interessen gegenüber Erwachsenen (Politik, Lehrkräften, Vereinsverantwortlichen etc.) zu artikulieren und durchzusetzen sowie Entscheidungen auf höherer Ebene mitzugestalten. Für entsprechendes Handeln spielen sowohl das Wissen um Entscheidungsprozesse, die Erfahrungen von Machtstrukturen als auch die eigene Einflussnahme auf Entscheidungsprozesse eine wichtige Rolle - alles bedeutsame Bausteine der politischen Bildung. Schließlich 
beinhaltet das freiwillige Engagement in vielen Bereichen die Erfahrung von Reziprozität, die Dewey als Grundlage sozialer Auffassungen und Interessen ansieht. Jugendliche sind in ihrem Engagement für sich und andere in soziale Kontexte und Konstellationen eingebunden, die unmittelbar auf sie zurückwirken und soziale Integrations- sowie Anerkennungsprozesse umfassen.

Somit finden sich im Engagement Jugendlicher prototypische Voraussetzungen des von Dewey konzipierten (demokratischen) Lernens durch Erfahrung, dem Lernen in und durch konkrete Handlungs- und Entscheidungssituationen - hierauf weisen auch die Analysen der Freiwilligensurveys hin, die die hohe Eigenmotivation und das hohe Interesse sowie die starke Eigentätigkeit ehrenamtlich engagierter Jugendliche betonen (vgl. Picot 2005). ${ }^{1}$

Eine stärker psychologisch orientierte Wendung des Erfahrungslernens präsentiert Reinders (2006). Hier untersucht er auf der Basis der Theorie gemeinnütziger Tätigkeit (vgl. Youniss \& Yates 1997; Reinders \& Youniss 2005), in welchem Umfang und wodurch vermittelt die politische Beteiligungsbereitschaft Jugendlicher durch Freiwilligenarbeit gefördert wird. Seine Theorie geht davon aus, dass soziales Engagement besonders während der Jugendphase zur Identitätsbildung beiträgt, da Jugendliche hier mit spezifischen, ihnen sonst fremd bleibenden Aspekten der Gesellschaft konfrontiert werden. Insbesondere Tätigkeiten, bei denen sie direkten Umgang mit bedürftigen Menschen haben, regen Jugendliche an, über soziale Ungleichheit und ihre Position in der Gesellschaft nachzudenken (ideology-Erfahrung). Hierdurch beginnen sie, ihr Selbstbild in Richtung auf ein höheres Maß an Prosozialität zu verändern. Wenn Jugendliche in ihrer Tätigkeit Erfolgserlebnisse haben und Effekte erzielen, machen sie Erfahrungen von Selbstwirksamkeit oder ,,agency“, was ihr prosoziales Verhalten wiederum verstärkt. Das Bewusstsein prosozialen Verhaltens stärkt die Bereitschaft, gesellschaftliche Verantwortung zu übernehmen und sich damit an demokratischen Prozessen zu beteiligen: „Heranwachsende werden im Rahmen ihres Engagements prosoziales Verhalten entwickeln, Erfahrungen von Selbstwirksamkeit machen (agency-Erfahrung) und sich mit Weltsichten auseinandersetzen (ideology-Erfahrung), die zu verstärkter politischer Beteiligungsbereitschaft führen" (Reinders 2006, S. 603).

Während aus den Deweyschen Überlegungen Beteiligungsbereitschaft - „Demokratie als Lebensform“ - als erlerntes Verhalten verstanden werden kann, das aus der erworbenen Fähigkeit zur politischen Partizipation und der in Ernstsituationen erlebten Notwendigkeit von politischer Beteiligung entsteht, betont das Modell von Reinders stärker prosoziale Einstellungen als Motor für politische Beteiligungsbereitschaft. ${ }^{2}$ Reinders zeigt auf der Basis von zwei unterschiedlichen empirischen Studien, dass es zwar auch einen direkten Effekt der Freiwilligenarbeit gibt: Freiwillig engagierte Jugendliche äußern insgesamt eine höhere Bereitschaft zu politischem Engagement als Nichtengagierte. Über die Einbeziehung der Konstrukte „Prosozialität“3, ,,agency-Erfahrung“ und ,ideologyErfahrung“ gelingt es ihm allerdings, die Erklärungskraft seiner Modelle deutlich zu erhöhen. Zugleich sinkt durch Einbeziehung dieser Variablen der direkte Zusammenhang zwischen freiwilligem Engagement und politischer Partizipationsabsicht deutlich. Reinders folgert daraus, dass diesen Variablen eine wesentliche Mediatorfunktion zukommt. Der stärkste Mediationseffekt ist nach seinen Analysen für das Konstrukt „Prosozialität“ nachzuweisen, ein vergleichsweise geringer für die ideology-Erfahrung. ${ }^{4}$ 


\section{Fragestellungen}

Im Folgenden soll den Effekten des ehrenamtlichen Engagements im Jugendalter auf das politische Engagement im Erwachsenenalter nachgegangen werden. Die Grundlage hierfür bildet eine Studie, in der aufgrund einer Retrospektivbefragung junger Erwachsener Daten vorliegen, die in einer Längsschnittperspektive auswertbar sind. Dabei wird in drei Teilschritten vorgegangen:

1. Zunächst wird geprüft, inwieweit freiwilliges Engagement in der Jugend die faktische politische Partizipation im Erwachsenenalter fördert. Hier wird an ein Desiderat angeknüpft, das Reinders selbst in seinem Artikel benennt: Da sich die von ihm verwendeten Daten auf Beteiligungsabsichten von Jugendlichen beziehen, entzieht sich ihm die Antwort, inwieweit diese Absichten ,auch in tatsächliches Verhalten umgesetzt“ (Reinders 2006, S. 614) werden.

2. In einem zweiten Schritt wird ein Ansatz von Reinders aufgenommen und untersucht, ob sich auch hier ein starker Effekt von „Prosozialität“ zeigt, der den direkten Effekt freiwilligen Engagements deutlich senkt.

3. In Erweiterung des Ansatzes von Reinders wird im dritten Schritt geprüft, inwiefern politisches Handeln mit spezifischen Lernerfahrungen im freiwilligen Engagement zusammenhängt. Hierbei wird davon ausgegangen, dass Jugendliche im freiwilligen Engagement neben der Herausbildung von Prosozialität und psychischen Dispositionen auch konkrete Fähigkeiten erwerben, die für späteres politisches Engagement entscheidend sind: Wenn in Alltagssituationen des freiwilligen Engagements die oben beschriebene Selbstwirksamkeit erlebt werden kann, so werden hier zugleich auch konkrete Handlungserfahrungen in Prozessen demokratischer Entscheidungsfindung und Interessenvertretung gemacht. Damit sind hier - neben der Herausbildung und Weiterentwicklung eines gesellschaftlichen Bewusstseins und von Prosozialität - der Erwerb von konkreten Handlungskompetenzen und Handlungswissen im Umgang mit sozialen und politischen Institutionen angelegt, die wesentliche Ressourcen für späteres Engagement und politische Beteiligung darstellen. Geht man nun davon aus, dass solche Kompetenzen und Wissen im freiwilligen Engagement erworben werden, so ergibt sich die Hypothese, dass der Erwerb dieses Wissens über das tatsächliche Handeln im Sinne von Dewey für späteres politisches Engagement genauso oder sogar bedeutsamer ist als Prosozialität.

\section{Empirische Analysen}

Im Folgenden werden die empirischen Analysen dargestellt, auf deren Grundlage die Frage nach den politischen Erträgen freiwilligen Engagements in der Jugend beantwortet werden sollen. Den Ausgangspunkt bilden dabei die von Reinders (2006) dargestellten Modelle. Hierbei ist allerdings zu beachten, dass die verwendete Datenbasis zwar eine Approximation, aber keine Eins-zu-Eins-Übertragung dieses Modells erlaubt. 


\subsection{Datengrundlage}

Die Datengrundlage dieser Untersuchung stellt ein standardisiert erhobener Datensatz dar, der im Rahmen des Projekts „Informelle Lernprozesse im Jugendalter in Settings des freiwilligen Engagements“ am Deutschen Jugendinstitut bearbeitet wurde. Dieses Projekt wurde im Rahmen des Forschungsverbunds Deutsches Jugendinstitut und Universität Dortmund durchgeführt und im April 2007 abgeschlossen. ${ }^{5}$

Die standardisierte Befragung richtete sich an erwachsene Personen im Alter zwischen 25 und 40 Jahren. Aus dieser Alterskohorte wurden 1.500 Personen, die vor ihrem 22. Lebensjahr mindestens ein Jahr freiwillig engagiert waren, retrospektiv zu ihren Lernerfahrungen, aber auch zu Einstellungen, Kompetenztransfers und aktuellem Engagement befragt. Zusätzlich wurden 552 Personen aus einer gleichaltrigen Vergleichsgruppe befragt, die in ihrer Jugend nicht ehrenamtlich engagiert war. Die Befragung wurde nach einem Pretest im Januar 2005 mit computerunterstützten telefonischen Interviews (CATI) in der Zeit von Mitte März 2005 bis Mitte Juni 2005 durchgeführt. Die Rücklaufquote von $48 \%$ liegt im Rahmen der üblichen Surveyforschung. Da die Stichprobe disproportional geschichtet war, d.h. die Ziehungswahrscheinlichkeit für Nichtengagierte deutlich geringer war als für in der Jugend Engagierte, wurden bei vergleichenden Analysen zum Ausgleich Designgewichte benutzt. Da es zudem wahrscheinlich ist, dass Personen, die bereits in der Jugendzeit politisch aktiv waren, auch später politisch aktiv sind, wurde die Gruppe der früher ,politisch Aktiven“ aus dem Sample herausgenommen. ${ }^{6}$ Auf diese Weise kann sichergestellt werden, dass zumindest für die Wirkung des freiwilligen Engagements in der Jugend auf politisches Engagement im Erwachsenenalter die zeitliche Ordnung von Ursache und Effekt (vgl. Prein 1998) gewahrt ist.

\subsection{Operationalisierungen}

Abhängige Variablen. Als abhängige Variablen wurden neben institutionellen auch nichtinstitutionelle Formen politischen Engagements untersucht, da insbesondere bei jungen Erwachsenen nicht davon ausgegangen werden kann, dass sich politische Partizipation nur in gesellschaftlich sanktionierten Formen vollzieht. Die Grundlage der Operationalisierung für beide Bereiche bildete der folgende Fragenblock: „Es gibt verschiedene Möglichkeiten, sich gesellschaftlich oder politisch einzusetzen. Ich nenne Ihnen jetzt einige Möglichkeiten. Sagen Sie mir doch bitte, welche davon in den letzten fünf Jahren auf Sie zutreffen. ${ }^{67}$ Aus jeweils drei der hier genannten Items wurden zwei Summenindizes gebildet, die die Guttman-Skalen darstellen.

- Der erste Index ,institutionelle Partizipationsformen“ umfasst die drei Items (in der Reihenfolge der Itemschwierigkeit): „Geldspenden an Parteien, Initiativen oder gemeinnützigen Organisationen“, „Mitgliedschaft in einer Partei“ und „Übernahme eines politischen Amtes“. Geldspenden werden als Form institutioneller gesellschaftlicher Beteiligung angesehen, die den geringsten Anforderungsgrad haben. Auch Parteimitgliedschaften verursachen Kosten, erfordern zusätzlich aber eine persönliche Positionierung sowie ggf. die Teilnahme an Sitzungen. Dieses Item hat somit die zweithöchste Schwierigkeit. 82,5\% der Mitglieder einer Partei geben an, Geld 
zu spenden. Die Übernahme eines politischen Amtes stellt gegenüber der - möglicherweise auch passiven - Parteimitgliedschaft nochmals eine Steigerung des gesellschaftlichen Engagements dar. Dies zeigt sich auch empirisch: 89,2\% der Personen, die ein Amt übernommen haben, geben auch Spenden. Erstaunlich - da Alltagstheorien widersprechend - ist die Tatsache, dass der Anteil der Amtsinhaber, die auch Mitglieder einer Partei sind, nur 66,2\% beträgt. Dennoch hat dieser Summenindex einen Reproduzierbarkeitskoeffizienten von 0,983 , sodass er den gängigen methodischen Standards entspricht.

- Der zweite Index „nichtinstitutionelle Beteiligungsformen“ umfasst die drei Items „Beteiligung an Unterschriftenaktionen“, „Beteiligung an Demonstrationen“ und „Beteiligung an politischen Aktionen wie Besetzungen oder Blockaden“. Während Unterschriftenaktionen als vergleichsweise unverbindlich angesehen werden können, liegen der persönliche Aufwand sowie mögliche Risiken bei Demonstrationen deutlich höher: 87,7\% der Demonstrationsteilnehmer/-innen beteiligen sich auch an Unterschriftenaktionen. Das persönliche Risiko und der Aufwand werden bei Aktionen wie Besetzungen oder Blockaden noch gesteigert. Von allen Personen, die sich an solchen Aktionen beteiligen, geben $85 \%$ auch die Beteiligung an Unterschriftenaktionen und 88,3\% die an Demonstrationen an. Auch bei diesem Index liegt der Reproduzierbarkeitskoeffizient mit einem Wert von 0,976 im akzeptablen Bereich.

Beide Indizes basieren auf retrospektiv erhobenen Informationen, wie sie im Bereich der Lebensverlaufsforschung üblich sind. Deren Validität kann - insbesondere aufgrund von falscher Erinnerung - problematisch sein. Dies betrifft allerdings weniger die Items mit höherer Itemschwierigkeit, zeigt doch die Gedächtnispsychologie, „,dass Ereignisse oder Ereignissequenzen, die lange andauern, emotional bedeutsam oder folgenschwer sind, eine höhere Wahrscheinlichkeit haben, korrekt abgerufen zu werden als solche, die kurz andauern, emotional neutral sind und keine merklichen Folgen für den Erinnernden haben." (Matthes, Reimer \& Künster 2007, S. 72). Inwieweit somit Spenden oder die Beteiligung an Unterschriftenaktionen richtig erinnert werden, kann vor diesem Hintergrund problematisiert werden. Allerdings kommen vergleichbare Analysen des Datensatzes auf der Grundlage der Einzelitems insgesamt zu identischen Ergebnissen (vgl. Düx et al. 2008, S. 335 ff.), sodass nicht davon ausgegangen werden muss, dass die präsentierten Ergebnisse auf reinen Artefakten beruhen.

Unabhängige Variable. Als unabhängige Variable wurde geprüft, ob die Befragten vor dem 22. Lebensjahr nach eigenen Angaben mindestens ein Jahr lang freiwillig engagiert gewesen sind (im weiteren Text: „Engagement“"). Diese Angabe, die als Dummy-Variable in das Modell einbezogen wurde, verweist im Sinne der vorgestellten theoretischen Überlegungen auf Lernerfahrungen durch Engagement, auf vorgängige Lernprozesse im und durch das ehrenamtliches Engagement. Mit der anfangs skizzierten Fragestellung wurde angenommen, dass diese (Lern-)Erfahrungen maßgeblichen Einfluss auf späteres politisches Engagement haben.

Kontrollvariablen. Da freiwilliges Engagement systematisch mit dem soziodemografischen Hintergrund einer Person variiert (vgl. etwa Konsortium Bildungsberichterstattung 
2006, S. 65), wurden das Alter, das Geschlecht, die Herkunft aus der ehemaligen DDR, der Migrationshintergrund sowie die Anzahl der Bildungsjahre systematisch kontrolliert. Als Personen mit Migrationshintergrund wurden alle Befragten angesehen, die entweder nicht in Deutschland geboren waren oder bei denen nicht beide Eltern in Deutschland geboren waren oder in deren Haushalt nicht nur Deutsch die Verkehrssprache war. Der Bildungshintergrund wurde auch mit alternativen Konzepten erfasst (bspw. über die CASMIN-Skala), da sich hierdurch keine wesentlichen Änderungen hinsichtlich der zentralen Aussagen dieses Beitrags ergaben, wurde der Operationalisierung über „Bildungsjahre“ aus Platzgründen Vorrang gegeben.

Mediatoren. Ein wesentlicher intervenierender Faktor in dem von Reinders dargestellten Modell stellt „Prosozialität“ dar. In der hier verwendeten Studie steht keine so differenzierte Skala zur Erfassung solcher Einstellungen zur Verfügung. Auf der Grundlage der Frage „Wie wichtig sind folgende Dinge für Sie persönlich?“ wurde vereinfachend das Antwortitem „Sozial Benachteiligten und gesellschaftlichen Randgruppen helfen“ als Proxy-Variable genutzt. Die Antworten wurden auf einer 5-stufigen Skala erhoben; diese wurden auf Werte von 0 für ,unwichtig“ bis 4 für ,,außerordentlich wichtig“ rekodiert. Eine wesentliche Voraussetzung für das von Reinders vorgestellte Modell ist, dass freiwilliges Engagement zu mehr Prosozialität führt; nur wenn dies der Fall ist, erscheint dieses Modell überhaupt sinnvoll. Zur Prüfung wurden im vorliegenden Fall ordinale Probitmodelle geschätzt, die einen - wenngleich nicht übermäßig starken - Effekt des freiwilligen Engagements in der Jugend auf die Einschätzung der Wichtigkeit zeigten, sozial Benachteiligten und gesellschaftlichen Randgruppen zu helfen.

Darüber hinaus wurde ein Indikator für Erfahrungen mit organisatorischen und politiknahen Tätigkeitsinhalten im freiwilligen Engagement einbezogen (in weiteren Text: „Organisationserfahrungen“). Da hierzu nur in der Jugend engagierte Personen befragt wurden, muss dieser Effekt in der Art eines Interaktionseffekts mit der Variablen „,in der Jugend engagiert" interpretiert werden. Hierdurch sollte geprüft werden, ob es allgemeine Selbstwirksamkeitserfahrungen in der Freiwilligenarbeit sind, die politisches Engagement begünstigen, oder ob in bestimmten Settings spezifische Erfahrungen der Selbstwirksamkeit gemacht werden, die zugleich spezifische Ressourcen für gesellschaftliche Partizipation vermitteln. Der hierzu benutzte Summenindex basiert auf der Frage: „Was waren die Hauptinhalte Ihrer freiwilligen/ehrenamtlichen Tätigkeit in der eben genannten Organisation? Ich nenne Ihnen jetzt mögliche Tätigkeiten. Bitte sagen Sie mir zu jeder, ob dies zutrifft oder nicht. Ging es überwiegend um ..." und fasst die Nennung der dichotom (,Ja, trifft $\mathrm{zu}^{\prime \prime}$ und „Nein, trifft nicht $\mathrm{zu}^{\circ)}$ ) kodierten sieben Items „um die Organisation und Durchführung von Veranstaltungen“, „um Interessenvertretung und Mitsprache in Ausschüssen und Gremien“, „um die Aufarbeitung fachbezogener oder politischer Themen“, „um Verwaltungstätigkeiten“, „um Informations- und Öffentlichkeitsarbeit“, „um Mittelbeschaffung, wie z.B. Spenden sammeln, Sponsoren ansprechen“ und „um Beratung“ zusammen. Zwar liegt die Reliabilität des Index (mit Cronbachs $\alpha=0,62$ ) unterhalb der vielfach genannten Schwelle von 0,7, dennoch wurde er für die folgenden Analysen genutzt, da er hoch mit Tätigkeitstypen korrespondiert, die auf der Basis aller Items gebildet worden waren. ${ }^{8}$ Diese sind wiederum stark korreliert mit spezifischen Lernerfahrungen im freiwilligen Engagement. Zur Vereinfachung der Interpretation wurde der 
Tab. 1: Ausmaß institutioneller und nicht-institutioneller Partizipation nach Engagement in der Jugend (in \%, Daten gewichtet, ohne in der Jugend politisch Aktive)

\begin{tabular}{|c|c|c|c|c|c|c|}
\hline \multirow{3}{*}{$\begin{array}{l}\text { Ausmaß der politischen } \\
\text { Partizipation in den } \\
\text { letzten } 5 \text { Jahren }\end{array}$} & \multicolumn{3}{|c|}{ Institutionell } & \multicolumn{3}{|c|}{ Nicht-institutionell } \\
\hline & \multicolumn{2}{|c|}{$\begin{array}{l}\text { In der Jugend } \\
\text { engagiert }\end{array}$} & \multirow[t]{2}{*}{ Insgesamt } & \multicolumn{2}{|c|}{$\begin{array}{l}\text { In der Jugend } \\
\text { engagiert }\end{array}$} & \multirow[t]{2}{*}{ Insgesamt } \\
\hline & Nein & $\mathbf{J a}$ & & Nein & $\mathbf{J a}$ & \\
\hline $0=$ gering & 40,5 & 28,5 & 38,0 & 38,0 & 29,7 & 36,3 \\
\hline 1 & 57,1 & 65,7 & 58,8 & 44,2 & 48,5 & 45,1 \\
\hline 2 & 2,3 & 4,4 & 2,7 & 16,9 & 19,7 & 17,5 \\
\hline $3=$ stark & 0,2 & 1,5 & 0,4 & 0,8 & 2,1 & 1,1 \\
\hline $\mathrm{N}$ (ungewichtet) & \multicolumn{2}{|c|}{1.932} & \multicolumn{4}{|c|}{1.930} \\
\hline
\end{tabular}

Quelle: Forschungsverbund DJ//Universität Dortmund - Informelle Lernprozesse

Index auf zwei Kategorien reduziert, die jeweils etwa 50\% der engagierten Jugendlichen umfassen. Die Kategorie, die als Dummy-Variable in das Modell aufgenommen wird, ist die mit höherer Organisationserfahrung; d.h. geringe Organisationserfahrung bildet die Referenzkategorie.

Bivariate Analysen. Betrachtet man im ersten Schritt zunächst nur die beiden abhängigen Variablen in Abhängigkeit vom Engagement in der Jugend (s. Tab. 1), so zeigt sich bereits hier, dass früher Engagierte sowohl hinsichtlich der institutionellen Partizipation, als auch (wenngleich etwas schwächer) hinsichtlich der nicht-institutionellen Partizipation aktiver sind als Personen, die in der Jugend nicht freiwillig engagiert waren.

Da sich hinter diesem Effekt allerdings auch Effekte von Drittvariablen wie Bildung, Herkunft oder Geschlecht verbergen könnten, wurde dieser Zusammenhang in einem folgenden Schritt durch ein multivariates Modell überprüft.

Modellschätzung. Da die abhängigen Variablen ordinalskaliert sind, wurde statt linearer Regressionsmodelle auf ordinale Probitmodelle zurückgegriffen. ${ }^{9}$ Die für die unabhängigen Variablen geschätzten Koeffizienten sind analog zur linearen Regression so zu lesen, dass ein positiver Wert die Wahrscheinlichkeit für ein stärkeres Engagement erhöht und ein negativer sie senkt.

Es wurden für jeden der beiden Indikatoren jeweils drei Modelle geschätzt: In einem ersten Schritt wurde geprüft, inwieweit freiwilliges Engagement in der Jugend die faktische politische Partizipation im Erwachsenenalter fördert. Hierzu wurde neben den Kontrollvariablen die Variable „Engagement“" in die Modelle einbezogen (s. Modelle 1 und 4 in Tab.2). Im zweiten Schritt wird untersucht, ob es auch bei faktischer Partizipation einen starken Effekt von „Prosozialität“" gibt, der den direkten Effekt von „Engagement“ senkt (s. Modelle 2 und 5). Im dritten wurden außerdem „Organisationserfahrung“ für in der Jugend engagierte Personen hinzugenommen, um zu prüfen, inwieweit der Erwerb von konkreten Handlungskompetenzen und Handlungswissen für spätere politische Beteiligung wichtig sind (s. Modelle 3 und 6 in Tab. 2). 
Tab. 2: Ordinale Probitmodelle zur institutionellen und nicht-institutionellen politischen Partizipation (Daten gewichtet, ohne in der Jugend politisch Aktive, Koeffizienten, die mindestens auf dem 5\%-Niveau signifikant sind, fett)

\begin{tabular}{|c|c|c|c|c|c|c|c|c|c|c|c|c|}
\hline \multirow[t]{3}{*}{ Variablen } & \multicolumn{6}{|c|}{ institutionelle Partizipation } & \multicolumn{6}{|c|}{ nicht-institutionelle Partizipation } \\
\hline & \multicolumn{2}{|c|}{ Modell 1} & \multicolumn{2}{|c|}{ Modell 2} & \multicolumn{2}{|c|}{ Modell 3} & \multicolumn{2}{|c|}{ Modell 4} & \multicolumn{2}{|c|}{ Modell 5} & \multicolumn{2}{|c|}{ Modell 6} \\
\hline & $\beta$ & $\mathbf{p}$ & $\beta$ & $\mathbf{p}$ & $\beta$ & $\mathbf{p}$ & $\beta$ & $\mathbf{p}$ & $\beta$ & $\mathbf{p}$ & $\beta$ & $\mathbf{p}$ \\
\hline Schwelle 1 & 1,60 & 000 & 2,01 & ,000 & 2,01 &, 000 & 0,27 & ,499 & 0,53 & 201 & 0,53 & 201 \\
\hline Schwelle 2 & 3,86 & ,000 & 4,31 & 000 & 4,32 &, 000 & 1,57 & 000 & 1,84 &, 000 & 1,84 &, 000 \\
\hline Schwelle 3 & 4,67 &, 000 & 5,11 &, 000 & 5,12 & 000 & 3,00 &, 000 & 3,30 &, 000 & 3,30 &, 000 \\
\hline Alter (Jahre) & $\mathbf{0 , 0 3}$ & ,001 & $\mathbf{0 , 0 3}$ & 001 & $\mathbf{0 , 0 3}$ & 001 & $-0,01$ & ,398 & $-0,01$ &, 348 & $-0,01$ &, 347 \\
\hline Frau & $-0,06$ &, 512 & $-0,12$ & 220 & $-0,11$ & 240 & $-0,04$ & 656 & $-0,08$ & ,391 & $-0,08$ & ,410 \\
\hline $\begin{array}{l}\text { in der DDR } \\
\text { geboren }\end{array}$ & $-0,13$ &, 310 & $-0,15$ & ,242 & $-0,15$ & ,241 & 0,35 & ,003 & 0,35 & ,004 & 0,35 & ,004 \\
\hline $\begin{array}{l}\text { Migrationshin- } \\
\text { tergrund }\end{array}$ & $-0,15$ & ,207 & $-0,19$ & 103 & $-0,19$ & ,099 & $-0,13$ & ,284 & $-0,15$ & ,203 & $-0,15$ & ,201 \\
\hline Bildungsjahre & $\mathbf{0 , 0 7}$ &, 000 & $\mathbf{0 , 0 7}$ & ,000 & $\mathbf{0 , 0 7}$ &, 000 & $\mathbf{0 , 0 7}$ &, 000 & $\mathbf{0 , 0 7}$ &, 000 & $\mathbf{0 , 0 7}$ &, 000 \\
\hline $\begin{array}{l}\text { in der Jugend } \\
\text { engagiert }\end{array}$ & 0,25 & ,000 & 0,22 & ,002 & 0,06 & ,415 & 0,11 & ,089 & 0,08 & ,193 & 0,01 & ,928 \\
\hline Prosozialität & & & 0,18 & ,001 & 0,18 & ,001 & & & 0,12 &, 023 & 0,12 &, 025 \\
\hline $\begin{array}{l}\text { Organisations- } \\
\text { erfahrung }\end{array}$ & & & & & 0,29 &, 000 & & & & & 0,15 & ,029 \\
\hline $\mathrm{N}$ (ungewichtet) & 1.918 & & 1.910 & & 1.915 & & 1.916 & & 1.914 & & 1.91 & \\
\hline
\end{tabular}

Quelle: Forschungsverbund DJI/Universität Dortmund - Informelle Lernprozesse

Effekte der Kontrollvariablen. Durchgängig in allen Modellen ist ein Bildungseffekt und damit verbunden auch ein Schichteffekt. ${ }^{10}$ Höhere Schulbildung fördert sowohl institutionelle als auch nicht-institutionelle Partizipation. Bei der institutionellen Partizipation kommt ein Alterseffekt hinzu: Ältere Befragte sind eher politisch engagiert. Dieser Effekt ist bei nicht-institutioneller Partizipation nicht vorhanden. Schließlich gibt es Herkunftseffekte: Nicht-institutionelle Formen politischen Engagements sind stärker bei Befragten, die in der früheren DDR geboren sind, institutionelle Formen sind geringer ausgeprägt bei Personen mit Migrationshintergrund. Allerdings ist letzterer Effekt nicht auf dem 5\%Niveau signifikant.

Effekte der unabhängigen Variablen und Mediatorvariablen. Unter Kontrolle all dieser Parameter zeigt sich zunächst in den einfachen Modellen 1 und 4 ein Effekt des Engagements in der Jugend - bei nicht-institutionellen Partizipationsformen allerdings nur auf dem 10\%-Signifikanzniveau. Freiwilliges Engagement hat damit nachhaltige Effekte auf die politische Partizipation im Erwachsenenalter - und dies auch dann, wenn das Engagement in der Jugend nicht im Bereich der Politik stattgefunden hat.

In den Modellen 2 und 5 zeigt sich, dass sich der direkte Effekt des freiwilligen Engagements nicht wesentlich verringert, wenn Prosozialität kontrolliert wird. Der Effekt des Engagements ist bei nicht-institutionellen Partizipationsformen nun allerdings noch nicht einmal mehr auf dem 10\%-Niveau signifikant. Wenngleich also ein schwacher indirekter Effekt bestehen könnte, ist hier - anders als bei Reinders - keine deutliche Verminde- 
rung des direkten Effekts des freiwilligen Engagements sichtbar. Dies könnte einerseits daran liegen, dass in den hier vorliegenden Analysen nicht auf eine umfangreich getestete Skala, sondern auf eine Proxy-Variable zurückgegriffen wurde, andererseits aber auch daran, dass aktuelle Einstellungen und Meinungen stärker zusammenhängen als Einstellungen und Handeln.

Demgegenüber schwächt sich der Haupteffekt des Engagements sehr deutlich ab, wenn in den Modellen 3 und 6 ,Organisationserfahrung“ in das Modell aufgenommen wird. Der Effekt des Engagements verschwindet, der Effekt von „Prosozialität“ bleibt stabil, für beide Engagementformen zeigt sich ein starker Effekt spezifischer Handlungserfahrungen im freiwilligen Engagement.

Alle beschriebenen Effekte betreffen sowohl institutionelle als auch nicht-institutionelle Partizipationsformen, wobei die Effekte bei Letzteren allerdings insgesamt deutlich schwächer sind.

Da das Konzept der Varianzaufklärung im Rahmen gewichteter Probitanalysen methodisch fraglich ist, wurden exemplarisch für spezifische Konstellationen der Kovariablen die auf der Basis von Modell 3 geschätzten Anteile der jeweiligen Gruppe an den institutionellen Partizipationsformen berechnet. Dabei wurden die Variablen Engagement, Prosozialität und Organisationserfahrung variiert, alle anderen wurden konstant gehalten (Alter 30 Jahre, Geschlecht männlich, nicht in der DDR geboren, kein Migrationshintergrund, Universitätsabschluss). ${ }^{11}$

Hierbei zeigt sich zunächst, dass unter Kontrolle der anderen Variablen kaum Effekte vom Engagement per se ausgehen: Beträgt der geschätzte Anteil der Personen ohne institutionelles politisches Engagement bei Nichtengagierten $56 \%$, so geht er nur auf etwa 54\% herunter, wenn zwar Engagement, aber nur geringe Prosozialität und geringe Organisationserfahrung vorliegen. Demgegenüber hat Prosozialität einen sehr starken Effekt. So sinkt etwa bei Nichtengagierten der Anteil derer, die von keinerlei institutioneller Partizipation berichten, durch hohe Prosozialität auf 28\%, bei Engagierten auf 26\%. Zwar geringer, aber dennoch sehr deutlich ist der Effekt von Organisationserfahrungen: So sinkt auch hier bei Engagierten der Anteil derer, die über keinerlei institutionelle Partizipation berichten, von 54\% auf 42\%; kommen Prosozialität und Organisationserfahrung zusammen, sogar auf $18 \%$.

\section{Fazit}

Fasst man die dargestellten Überlegungen und Befunde zusammen, so spricht einiges für die These der Bedeutung des Lernens im freiwilligen Engagement. Aus den empirischen Analysen lassen sich mindestens vier Punkte festhalten, die die Analysen von Reinders ergänzen und auf die Relevanz von Lern- und Bildungsprozessen im freiwilligen Engagement hinweisen. So zeigen sich erstens - wie schon von Reinders vermutet - positive Effekte des freiwilligen Engagements in der Jugend nicht nur auf politische Beteiligungsabsichten in der Jugend, sondern auch auf die tatsächliche politische Partizipation im Erwachsenenalter.

Allerdings kann zweitens auf der Grundlage der vorgestellten Daten eine Mediatorfunktion von „Prosozialität“ in Bezug auf faktisches Handeln mit den analysierten Daten 
nicht nachgewiesen werden. Zwar hat Prosozialität einen sehr deutlichen Effekt auf die politische Partizipation und zudem besteht ein Zusammenhang zwischen Freiwilligenarbeit und Prosozialität. Jedoch nimmt der Effekt des freiwilligen Engagements nur geringfügig $a b$, wenn prosoziale Einstellungen kontrolliert werden. Somit kann diese Variable vor allem als unabhängiger Effekt interpretiert werden.

Die allgemeinen Selbstwirksamkeitserfahrungen (,agency“) konnten aufgrund der Datenlage nicht analog zum Modellansatz von Reinders kontrolliert werden. Allerdings zeigt sich drittens durch die Einführung des Effekts „Organisationserfahrung“, dass es spezifische Handlungserfahrungen im Engagement sind, die eine politische Partizipation fördern. Politisches Engagement ist damit - im Sinne der im Anschluss an Dewey entwickelten Hypothese - auch ganz wesentlich mit Lern- und Bildungsprozessen verbunden und nicht primär mit Einstellungsveränderungen. Dieser Befund lässt sich als eine Bestätigung der Überlegungen verstehen, dass das im konkreten Tun Erlebte und Erfahrene - die Fähigkeit zu und das Wissen um Beteiligung und Beteiligungsstrukturen - eine zentrale Bedeutung für spätere aktive politische Mitwirkung hat.

Betont werden kann viertens auch, dass die Effekte des freiwilligen Engagements bei nicht-institutionellen Beteiligungsformen generell deutlich schwächer sind als bei institutionellen. Gerade bei Letzteren sind Wissen um und Erfahrungen mit den Funktionsweisen von Organisationen notwendig, um kompetent agieren zu können. Auch dieser Befund stützt somit indirekt die Hypothese, dass die wesentliche Bedeutung des freiwilligen Engagements im ,learning by doing“, im „Demokratie lernen“ besteht und damit vor allem einen Bildungseffekt darstellt, durch den spezifische, für den Bereich des politischen Handelns notwendige Handlungskompetenzen aufgebaut werden.

Dies verweist in unseren Augen wieder auf die Bedeutung des Ehrenamtes als konkretes Lernfeld, in dem durch Erleben von Handeln und dessen Konsequenzen im Sinne Deweys gelernt wird und konkrete Kompetenzen erworben werden. Wenn vor allem die praktische Erfahrung ehrenamtlichen Tuns für weiteres Engagement bedeutsam ist und dies nicht primär über soziale Einstellungen vermittelt wird, so ist eine hohe Relevanz von Wissensund Kompetenzbeständen für weiteres ehrenamtliches Engagement anzunehmen.

Ohne hier systematisch Konsequenzen für Schule oder Jugendarbeit ableiten zu können, ist dieser Befund auch für diese Bildungseinrichtungen und -angebote ein Hinweis auf die Relevanz „echter“ Beteiligung und die Bedeutung von „Ernstsituationen“. Vor dem Hintergrund der vorliegenden Befunde wäre somit kritisch zu prüfen, inwieweit „Übungsplätze“ für Beteiligungsprozesse entsprechende Lernprozesse tatsächlich initiieren können. Schule und Jugendarbeit wären damit herausgefordert, Lernerfahrungen in Ernstsituationen zu ermöglichen und echte Beteiligung - unter Abgabe von Verantwortung an Kinder und Jugendliche - zuzulassen und zu fördern.

Demokratie will, so könnte man es auf den Punkt bringen, gelernt sein. Es reicht offensichtlich nicht aus, im Engagement etwas erlebt zu haben, was das Empfinden von Selbstwirksamkeit verändert. Diese Erfahrung machen freiwillige Feuerwehrleute, die einen Brand gelöscht haben, oder Fußballtrainer, deren Verein ein entscheidendes Spiel gewonnen hat, sicher auch. Zum politischen Handeln führt dieses Erleben nicht unbedingt. Vielmehr zeigt sich hier, dass das gesellschaftliche Engagement umso stärker ist, je mehr in der Freiwilligenarbeit Tätigkeiten ausgeführt wurden, über die konkrete Fähigkeiten für ein Engagement im Bereich der Politik vermittelt werden. 


\section{Anmerkungen}

1 Diese Idee wird derzeit verstärkt als Service Learning, als Lernen im Dienste für andere und für biographisches Lernen auch systematisch in formale Bildungssysteme wie die Schule integriert (vgl. Sliwka 2004).

2 Hingewiesen werden muss mit Bezug auf das Modell von Reinders auch, dass das ehrenamtliche Engagement Jugendlicher (im Jugendverband, in der Schule, in Vereinen) nicht zwingend mit der Erfahrung im Umgang „mit bedürftigen Menschen“ verbunden ist. Oftmals beziehen sich die Formen des Engagements in Vereinen oder Jugendverbänden auf jugendliche Selbstorganisation in Freizeitaktivitäten, Organisation von Festen und Konzerten oder eigenständige Leistungsbereiche (Lebensrettung, Naturprojekte etc.) (vgl. Düx et al. 2008; Züchner 2006).

3 „Prosozialität“ operationalisiert Reinders durch eine aus der US-amerikanischen Forschung übernommene Skala mit sechs Items. Diese zielen auf geäußerte Handlungsabsichten in hypothetischen Situationen ab und stellen damit eher eine Erfassung prosozialer Einstellungen dar.

4 Ob hieraus abgeleitet werden kann, dass „Prosozialität“ eine intervenierende Variable ist, wird allerdings von Reinders problematisiert, da bei den vorliegenden Daten keine zeitliche Ordnung hergestellt wird: Somit ist nicht feststellbar, ob sich prosozial eingestellte Personen stärker freiwillig engagieren (Selektionseffekt) oder freiwilliges Engagement prosoziales Verhalten fördert (Intervention).

5 Das Projekt wurde vom Bundesministerium für Familie, Senioren, Frauen und Jugend (BMFSFJ) sowie vom Ministerium für Generationen, Familie, Frauen und Integration des Landes Nordrhein-Westfalen (MGFFI) kofinanziert. Es begann mit einer Explorationsphase von Januar 2003 bis Oktober 2003; die Hauptphase lief von November 2003 bis April 2007. Neben der hier dargestellten standardisierten Untersuchung wurden umfangreiche qualitative Analysen durchgeführt (vgl. Düx et al. 2008).

6 Als in der Jugend politisch aktiv wurden dabei alle Personen gewertet, die in Gewerkschaften, Parteien, deren Jugendorganisationen, einem politiknahen Jugendverband wie der SJDDie Falken, bei Greenpeace, Amnesty International, Attac oder in der Antifa engagiert waren. Diese Gruppe umfasst 113 Personen.

7 Obwohl der genannte 5-Jahreszeitraum für jüngere Befragte nicht unbedingt überlappungsfrei mit dem freiwilligen Engagement in der Jugend ist, wurde die untersuchte Gruppe bei den hier vorgestellten Analysen nicht um die jüngeren Altersjahrgänge bereinigt, da davon auszugehen ist, dass eher Ereignisse jüngeren Datums erinnert werden. Zudem wurde in multivariaten Analysen u.a. aus diesem Grund die Variable Alter kontrolliert.

8 Hierzu wurde über alle Items eine Clusteranalyse durchgeführt, auf deren Basis vier Tätigkeitstypen identifiziert wurden (vgl. Düx et al. 2008). Diese wurden in Anlehnung an eine Typologie aus der qualitativen Analyse als ,praktische Helfer/in“, „Gruppenleiter/in oder Trainer/in“, „Organisator/in“ und „Funktionär/in“ bezeichnet. Lerneffekte im Rahmen freiwilligen Engagements zeigen sich dabei vor allem bei den beiden letztgenannten Gruppen.

9 Probitmodelle sind ein Verfahren zur multivariaten Regressionsanalyse bei kategorialen abhängigen Variablen (zur Übersicht vgl. Tutz 2000, S. 122 ff.). Bei diesen würden im Falle der Anwendung der linearen Regression wesentliche Anwendungsvoraussetzungen verletzt. Probitmodelle basieren auf der Annahme, dass die beobachtete abhängige Variable die Vereinfachung einer normalverteilten metrischen Variablen darstellt. Hat die abhängige Variable nur zwei Kategorien, die mit den Werten 0 und 1 kodiert sind, wird mit einem binären Probitmodell die Wahrscheinlichkeit für die Ausprägung 1 geschätzt - bspw. die Wahrscheinlichkeit, ein politisches Amt zu übernehmen. Ordinale Probitmodelle (vgl. Tutz 2000, S. 205 ff.) erweitern diesen Ansatz, wenn die abhängige Variable mehr als zwei Kategorien hat, die zudem als geordnet angesehen werden, wie hier das Ausmaß politischen Engagements. Hier 
werden auf der Grundlage des binären Modells die Wahrscheinlichkeiten geschätzt, jeweils bestimmte Schwellen zu überschreiten. Hier liegt bspw. eine erste Schwelle zwischen „keinem Engagement" einerseits und allen Formen des Engagements andererseits (also mindestens „Geldspenden“), eine zweite zwischen „keinem Engagement oder Geldspenden“ einerseits und „Parteimitgliedschaft oder Amtsübernahme“ andererseits etc. Dabei ist eine wesentliche Modellannahme, dass sich Modellparameter mit Ausnahme der jeweiligen Konstanten nicht unterscheiden. Diese unterschiedlichen Konstanten werden in der Darstellung hier als „Schwelle 1“ bis „Schwelle 3“ bezeichnet. Der Parameter „Schwelle 1“ kennzeichnet dabei die Konstante für den Übergang von ,politisch nicht aktiv“ "zu mindestens geringer politischer Partizipation (Geldspenden bzw. Unterschriften), die Schwellen 2 und 3 jeweils die Konstanten für höhere Partizipationsniveaus.

10 Dieser Effekt tritt auch dann auf, wenn statt Bildungsjahren bspw. das Berufsprestige kontrolliert wird oder unterschiedliche Schulabschlüsse als einzelne Parameter in das Modell genommen werden.

11 Die hier dargestellten Werte stellen Schätzungen dar, die mit Mess- und Stichprobenfehlern behaftet sind - aus diesem Grunde sollten sie als Anhaltspunkte gesehen und nicht als Punktschätzungen missverstanden werden.

\section{Literatur}

Autorengruppe Bildungsberichterstattung (2008). Bildung in Deutschland 2008. Ein indikatorengestützter Bericht mit einer Analyse zu Übergängen im Anschluss an den Sekundarbereich I. Bielefeld: W. Bertelsmann.

Auerbach, S. \& Wiedemann, U. (1997). „Jugend ohne Amt und Ehre?“ Eine Untersuchung zu Determinanten ehrenamtlichen Engagements Jugendlicher im kleinstädtischen Milieu. Pfaffenweiler: Centaurus.

Baumert, J., Klieme, E., Neubrand, M., Prenzel, M., Schiefele, U., Schneider, W. et al. (Hrsg.) (2001). PISA 2000 - Basiskompetenzen von Schülerinnen und Schülern im internationalen Vergleich. Opladen: Leske + Budrich.

Brenner, G. (2003). Bildung in Jugendarbeit und Schule. deutsche jugend, 51(7/8), 344-355.

Buhl, M. \& Kuhn, H.-P. (2005). Erweiterte Handlungsräume im Jugendalter: Identitätsentwicklung im Bereich gesellschaftlichen Engagements. In B. Schuster, H.-P. Kuhn \& H. Uhlendorf (Hrsg.), Entwicklung in sozialen Beziehungen - Heranwachsende in ihrer Auseinandersetzung mit Familie, Freunden und Gesellschaft (S. 217-237). Stuttgart: Lucius \& Lucius.

Bundesministerium für Familie, Senioren, Frauen und Jugend (Hrsg.) (2000). Freiwilliges Engagement in Deutschland - Freiwilligensurvey 1999 - Ergebnisse der Repräsentativerhebung zu Ehrenamt, Freiwilligenarbeit und bürgerschaftlichem Engagement. Band 1-3. Stuttgart: Kohlhammer.

Bundesministerium für Familie, Senioren, Frauen und Jugend \& Statistisches Bundesamt (Hrsg.) (2003). Wo bleibt die Zeit? Die Zeitverwendung der Bevölkerung in Deutschland 2001/02. http://www.destatis.de/jetspeed/portal/cms/Sites/destatis/Internet/DE/Content/Publikationen/ Fachveroeffentlichungen/Bevoelkerung/Fachveroebevoelkzeitverwendung,property=file.pdf. Zugriff am 08. Mai 2009.

Corsa, M. (1998). Jugendliche, das Ehrenamt und die gesellschaftspolitische Dimension. Recht der Jugend und des Bildungswesens, 46(3), 322-334.

Corsa, M. (2003). Jugendarbeit und das Thema „Jugendarbeit und Schule“ - aufgezwungen, nebensächlich oder existenziell. deutsche jugend, 51(9), 369-379. 
Dewey, J. (2000). Demokratie und Erziehung. Eine Einleitung in die philosophische Praxis. Weinheim: Beltz.

Dohmen, G. (2001). Das Informelle Lernen. Die internationale Erschließung einer bisher vernachlässigten Grundform menschlichen Lernens für das lebenslange Lernen aller. Bonn: Bundesministerium für Bildung und Forschung.

Düx, W. (1999). Das Ehrenamt im Jugendverband. Ein Forschungsbericht. Frankfurt a.M.: Dt. Verein f. öffentl. u. priv. Fürsorge.

Düx, W., Prein, G., Sass, E., \& Tully, C. (2008). Kompetenzerwerb im freiwilligen Engagement. Eine empirische Studie zum informellen Lernen im freiwilligen Engagement. Wiesbaden: VS Verlag für Sozialwissenschaften.

Enquete-Kommission „Zukunft des bürgerschaftlichen Engagements“ des Deutschen Bundestags (2002). Bericht - Bürgerschaftliches Engagement: auf dem Weg in eine zukunftsfähige Bürgergesellschaft, Schriftenreihe Band 4. Opladen: Leske + Budrich.

Faulstich, P. (2005). Lernen Erwachsener in kritisch-pragmatischer Perspektive. Zeitschrift für Pädagogik, 51, 528-542.

Fauser, K., Fischer, A., \& Münchmeier, R. (2006). Jugendliche als Akteure im Verband. Ergebnisse einer empirischen Untersuchung der Evangelischen Jugend. Jugend im Verband 1. Opladen: Barbara Budrich.

Fischer, C. (2001). „Das gehört jetzt irgendwie zu mir“. Mobilisierung von Jugendlichen aus den neuen Bundesländern zum Engagement in einem Umweltverband. Eine explorative Studie am Beispiel der BUNDjugend. Dissertation TU Chemnitz. http://archiv.tu-chemnitz.de/ pub/2002/0013. Zugriff am 08. Mai 2009.

Furtner-Kallmünzer, M., Hössl, A., Janke, D., Kellermann, D., \& Lipski, J. (2002). In der Freizeit für das Leben lernen. Eine Studie zu den Interessen von Schulkindern. Opladen: Leske + Budrich.

Gaskin, K., Smith, J. D., \& Paulwitz, I. (Hrsg.) (1996). Ein neues bürgerschaftliches Europa. Eine Untersuchung zur Verbreitung von Volunteering in zehn Ländern. Freiburg i. Br.: Lambertus.

Gensicke, T., Picot, S., \& Geiss, S. (2006). Freiwilliges Engagement in Deutschland 1999-2004. Ergebnisse der repräsentativen Trenderhebung zu Ehrenamt, Freiwilligenarbeit und bürgerschaftlichem Engagement. Wiesbaden: VS Verlag für Sozialwissenschaften.

Gerzer-Sass, A., Reupold, A., \& Nußhart, C. (2006). Kompetenzbilanz aus Freiwilligen-Engagement. München: DJI. http://www.dji.de/5_kompetenznachweis/KB_Kompetenzbilanz_ 281206.pdf. Zugriff am 08. Mai 2009.

Gille, M., Sardei-Biermann, S., Gaiser, W., \& de Rijke, J. (2006). Jugendliche und junge Erwachsene in Deutschland. Lebensverhältnisse, Werte und gesellschaftliche Beteiligung 12- bis 29Jähriger (Schriften des Deutschen Jugendinstituts: Jugendsurvey 3). Wiesbaden: VS Verlag für Sozialwissenschaften.

Hofer, M. (1999). Community service and social cognitive development in German adolescents. In M. Yates \& J. Youniss (Eds.), Roots of civic identity. International perspectives on community service and activism in youth (pp. 114-134). Cambridge: Cambridge University Press.

Hofer, M. \& Buhl, M. (2000). Soziales Engagement Jugendlicher: Überlegungen zu einer technologischen Theorie der Programmgestaltung. In H. P. Kuhn, H. Uhlendorf, \& L. Krappmann (Hrsg.), Sozialisation zur Mitbürgerlichkeit (S. 95-111). Opladen: Leske + Budrich.

Hurrelmann, K. \& Albert, M. (Hrsg.) (2002). Jugend 2002. 14. Shell Jugendstudie. Frankfurt a.M.: Fischer.

Hurrelmann, K. \& Albert, M. (Hrsg.) (2006). Jugend 2006. 15. Shell Jugendstudie: Eine pragmatische Generation unter Druck. Frankfurt a.M.: Fischer.

Jugendwerk der Deutschen Shell (Hrsg.) (1997). Jugend '97. Zukunftsperspektiven. Gesellschaftliches Engagement. Politische Orientierungen. Opladen: Leske + Budrich.

Jugendwerk der Deutschen Shell (Hrsg.) (2000). Jugend 2000. Opladen: Leske + Budrich. 
Konsortium Bildungsberichterstattung (2006). Bildung in Deutschland. Ein indikatorengestützter Bericht mit einer Analyse zu Bildung und Migration. Bielefeld: W. Bertelsmann.

Künemund, H. \& Schupp, J. (2007). Konjunkturen des Ehrenamts: Diskurse und Empirie. SOEPpapers on Multidisciplinary Panel Data Research 22. http://www.diw.de/deutsch/produkte/publikationen/soeppapers/docs/papers/diw_sp0022.pdf. Zugriff am 08. Mai 2009.

Lehmann, T. (2004). Jugendverbände und der Übergang in Arbeit. Vorstellung des Praxisforschungsprojekts ,Jugendverbände, Kompetenzentwicklung und biografische Nachhaltigkeit““. Sozialextra, 28(7/8), 32.

Lerner, R. M., Alberts, A. E., \& Bobek, D. L. (2007). Engagierte Jugend-lebendige Gesellschaft. Möglichkeiten zur Stärkung von Demokratie und sozialer Gerechtigkeit durch positive Jugendentwicklung. Bielefeld: Bertelsmann-Stiftung.

Matthes, B., Reimer, M., \& Künster, R. (2007). Techniken und Werkzeuge zur Unterstützung der Erinnerungsarbeit bei der computergestützten Erhebung retrospektiver Längsschnittdaten. Methoden - Daten - Analysen, 1(1), 69-92.

Münchmeier, R., Otto, H.-U., \& Rabe-Kleberg, U. (Hrsg.) (2002). Bildung und Lebenskompetenz. Kinder- und Jugendhilfe vor neuen Aufgaben. Opladen: Leske + Budrich.

Nohl, A.-M. (2001). Qualitative Bildungsforschung und Pragmatismus. Zeitschrift für Erziehungswissenschaft, 4, 605-623.

Oelkers, J. (2000). Demokratie und Bildung. Über die Zukunft eines Problems. Zeitschrift für Pädagogik, 46, 333-347.

Oesterreich, D. (2002). Politische Bildung von 14-Jährigen in Deutschland. Studien aus dem Projekt Civic Education. Opladen: VS Verlag für Sozialwissenschaften.

Olk, T. (2003). Bürgerschaftliches Engagement. Eckpunkte einer Politik der Unterstützung freiwilliger und gemeinwohlorientierter Aktivitäten in Staat und Gesellschaft. Neue Praxis, 33(3/4), $306-325$.

Oser, F. \& Biedermann, H. (Hrsg.) (2003). Jugend ohne Politik. Ergebnisse der IEA Studie politischem Wissen, Demokratieverständnis und gesellschaftlichem Engagement von Jugendlichen in der Schweiz im Vergleich mit 27 anderen Ländern. Zürich: Rüegger.

Otto, H.-U.\& Rauschenbach, T. (Hrsg.) (2004). Die andere Seite der Bildung. Zum Verhältnis von formellen und informellen Bildungsprozessen. Wiesbaden: VS Verlag für Sozialwissenschaften.

Picot, S. (2005). Freiwilliges Engagement Jugendlicher im Zeitvergleich 1999-2004. In T. Gensicke, S. Picot, \& S. Geiss, Freiwilliges Engagement in Deutschland 1999-2004. Ergebnisse der repräsentativen Trenderhebung zu Ehrenamt, Freiwilligenarbeit und bürgerschaftlichem Engagement (S. 202-258). München: VS Verlag für Sozialwissenschaften.

Prein, G. (1998). Modeling rational action: A longitudinal approach. In H.-P. Blossfeld \& G. Prein (Eds.), Rational Choice Theory and Large-Scale Data Analysis (pp. 247-257). Boulder: Westview Press.

Putnam, R. D. (1993). Making democracy work: Civic traditions in modern Italy. Princeton: Princeton University Press.

Putnam, R. D. (2000). Bowling alone. The collapse and revival of American community. New York: Simon \& Schuster.

Reichwein, S. \& Freund, T. (1992). Jugend im Verband: Karrieren-Action-Lebenshilfe. Opladen: Leske + Budrich.

Reinders, H. (2005). Jugend. Werte. Zukunft. Wertvorstellungen, Zukunftsperspektiven und soziales Engagement im Jugendalter (Schriftenreihe der Landesstiftung Baden-Württemberg 14). Stuttgart: Landesstiftung Baden-Württemberg. http://www.landesstiftung-bw.de/publikationen/files/sr-14_studie_jugendwertezukunft2.pdf. Zugriff am 08. Mai 2009.

Reinders, H. (2006). Freiwilligenarbeit und politische Engagementbereitschaft in der Adoleszenz. Skizze und empirischen Prüfung einer Theorie gemeinnütziger Tätigkeit. Zeitschrift für Erziehungswissenschaft, 9, 599-616. 
Reinders, H. \& Youniss, J. (2005). Community service and civic development in adolescence. Theoretical considerations and empirical evidence. In A. Sliwka, M. Diedrich, \& M. Hofer (Eds.), Citizenship education. Theory, research, practice (pp. 112-127). Münster: Waxmann.

Richter, H., Jung, M., \& Riekmann, W. (2006). Jugendverbandsarbeit in der Großstadt. Perspektiven für Mitgliedschaft und Ehrenamt am Beispiel der Jugendfeuerwehr Hamburg. Hamburg: Jugendfeuerwehr.

Schwab, J. (2006). Bildungseffekte ehrenamtlicher Tätigkeit in der Jugendarbeit. deutsche jugend, $54(7 / 8), 320-328$.

Sliwka, A. (2004). Service Learning: Verantwortung lernen in Schule und Gemeinde. Beiträge zur Demokratiepädagogik (Schriftenreihe des BLK-Programms „Demokratie lernen \& leben“, hrsg. von W. Edelstein \& P. Fauser). http://www.blk-demokratie.de/fileadmin/public/dokumente/Sliwka.pdf. Zugriff am 08. Mai 2009.

Thole, W. \& Hoppe, J. (Hrsg.) (2003). Freiwilliges Engagement - ein Bildungsfaktor. Berichte und Reflexionen zur ehrenamtlichen Tätigkeit von Jugendlichen in Schule und Jugendarbeit. Frankfurt a.M.: Dt. Verein f. öffentl. u. priv. Fürsorge.

Tutz, G. (2000). Die Analyse kategorialer Daten. Anwendungsorientierte Einführung in LogitModellierung und kategoriale Regression. München: Oldenbourg.

Torney-Purta, J., Lehmann, R., Oswald, H., \& Schulz, W. (2001). Citizenship and education in twenty-eight countries. Civic knowledgement and engagement at age fourteen. Amsterdam: IEA.

Verba, S., Schlozman, K. L., \& Brady H. E. (1995). Voice and equality: Civic volunteerism in American politics. Cambridge: Harvard Univ. Press.

Yates, M. \& Youniss, J. (1996). Communitiy service and political-moral identity in adolescents. Journal of Research on Adolescence, 6(3), 271-284.

Youniss, J., Mc Lellan, J. A., \& Yates, M. (1997). What we know about engendering civic identity. American Behavioural Scientist, 40, 620-631.

Youniss, J. \& Yates, M. (1997). Community service and social responsibilitiy in youth. Chicago: Univ. of Chicago Press.

Züchner, I. (2006). Mitwirkung und Bildungseffekte in Jugendverbänden - Ein empirischer Blick. deutsche jugend, 54(5), 201-209. 